\title{
DEVELOPING TIME-SENSITIVE HYPERTEXT LINKING AND NAVIGATION SUPPORT
}

\author{
Anneli Heimbürger \\ Tampere University of Technology, Pori, Advanced Multimedia Center (AMC)
}

\begin{abstract}
Hypertext links play an important role in tracking the relationships within and among sets of documents. In our paper we discuss two approaches to creating dynamic workflow charts with time-sensitive linking and navigation support for training time-based project management in distributed Web-based environments. A bottom-up approach is based on the XML Linking Language (XLink) and the top-down approach is based on the XML Topic Maps (XTM) 1.0 specification.
\end{abstract}

Key words: design technology, hypermedia, industry practices, Internet

\section{INTRODUCTION}

Web-based project and document management platforms play an important role in distributed project execution. Particularly when projects involve numerous teams situated in various locations, such platforms provide an effective place and time independent environment for communication, information interchange and monitoring the progress of the project. Before implementing such a system, an organization analyses its information management processes with related teams, project deliverables and document process steps. As a result of the analysis, the organization has defined document classification, uses, user groups, life cycles, states (such as new - in work - for approval - published), structure of project portfolios and finally, a flowchart of the whole process. The organization-specific process flowchart is an essential tool for training project teams, members and managers in organizations. The chart makes it easier for project members to understand their roles and responsibilities in a project. They can see 
themselves as parts in a whole workflow. This often helps project teams to adopt a new project management system and new ways of action, adds commitment and minimizes possibly existing resistance to change.

However, mapping training project operations against the project schedule is a problem with a static flowchart. Project management instructors need additional methods and tools to illustrate temporal characteristics of a project and to create time-based views to project repositories. The main object of training time-based project management in enterprises is to decrease project lead-times and bring economical benefits with increased competitiveness.

In our paper we discuss two approaches to create dynamic workflow charts with time-based linking and navigation support. The bottom-up approach is based on the XML Linking Language (XLink) specification, which has a recommendation status in the World Wide Web Consortium (W3C). XLink provides richer and more flexible mechanisms for defining link traversal rules than traditional (X)HTML. The top-down approach is based on the XML Topic Maps (XTM) specification, which is the Web version of the ISO 13250 Topic Maps standard (ISO 13250; XTM TopicMaps.Org 2003).

The remainder of the paper is organized as follows. Related work is reviewed in Section 2. The concept of time-sensitive hypertext linking mechanism is introduced in Section 3. Bottom-up and top-down approaches to create dynamic workflow charts with time-based linking and navigation support are discussed in Section 4. Our conclusions and issues for further research are presented in Section 5.

\section{RELATED WORK}

Open hypermedia is an area that has been studied by the hypermedia community for several years and for which a number of systems have been implemented (Bieber et al. 1997; Carr et al. 2000; Carr et al. 2001; Grønbæk and Trigg 1999). In open hypermedia systems (OHS), links are managed and stored in special databases called link databases or linkbases. The idea of abstracting links from documents allows for a great deal of flexibility in link maintenance and re-use. The XML Linking Language (XLink) is increasingly moving the Web towards the open hypermedia approach (Heimbürger 2003; W3C 2003c). XLink is also a part of the W3C's Semantic Web -activity (Berners-Lee et al. 2001; van Ossenbruggen et al. 2002; W3C 2003a). The aim of the Semantic Web is to have data on the Web defined and linked in a way that it can be used by machines not just for 
display purposes, but for integration and reuse of information across various applications.

Ontologies and ontology representation languages are a popular research topic in various communities such as knowledge engineering, natural language processing, co-operative information systems, intelligent information integration, and knowledge management (Carr et al. 2001; Guarino 1995; RosettaNet 2003; WordNet 2003). Some authors have also dealt with time ontologies (Zhou and Fikes 2002). Applications of the Topic Maps standard include mainly designing Web pages or portals (Park and Hunting 2003; Pepper 2003; Pepper and Garshol 2003). Hameri et al. have investigated distributed project management and its training extensively, both from the general and time-critical point of view (Hameri, and Nihtilä, 1997; Hameri and Nihtilä 1998; Hameri and Heikkilä 2002).

Our study is a complete new effort to describe time-sensitive linking mechanisms, apply it to time-based project management and its training in distributed multi-organizational project environments.

\subsection{Time-Sensitive Linking Mechanisms}

Time, as an abstract concept, means a space of time points reached from one another by before and after-like operators. Time is a collection of temporal items such as, moments, durations and instants. Things in time are somehow correlated with these temporal items. We call this correlation temporality or temporal rules.

Time points are used to represent specific, instantaneous, points along a time line. Time intervals are a set of constraints between two points, a start and an end time. Relationships between time intervals are defined with Allen's 13 relations: before $\left(t_{1}, t_{2}\right)$, equal $\left(t_{1}, t_{2}\right)$, meets $\left(t_{1}, t_{2}\right)$, overlaps $\left(t_{1}\right.$, $\left.t_{2}\right)$, during $\left(t_{1}, t_{2}\right)$, starts $\left(t_{1}, t_{2}\right)$, finishes $\left(t_{1}, t_{2}\right)$, and their inverse (Allen 1991).

In time-sensitive Web applications we can identify the following levels: a) content and/or pieces of content are functions of time, b) content and/or pieces of content as well as links between them are functions of time and c) links between content and/or pieces of content are functions of time. Timesensitivity can include either predictable or non-predictable temporal relationships among content elements included in an application. In a predictable case, temporal rules can be resolved and defined before the application runs. Such an application consists of a single time line with which the different elements are synchronised. It is not affected by the activation of links and as such does not provide for non-predictable changes.

User interactions or other external entities that lead to non-linear proceeding or adaptation according to the user's interest are examples of non-predictable temporal relationships. Non-predictable temporal relations 
can be described with time-sensitive link traversals. Allen's interval relations give us a base for describing temporal rules and creating time-sensitive linking mechanisms and navigation support for time-based project workflow charts.

\section{FROM BOTTOM-UP TO TOP-DOWN APPROACH}

We can approach the problem of describing time-sensitive hypertext linking mechanisms in two ways: with the top-down and bottom-up approach. Extensible Markup Language (XML) is the common base for both (W3C 2003b). The top-down approach can be applied to describing both project specific and general temporal rules whereas the bottom-up approach concentrates on describing project specific temporal rules.

There are organizations with only one type of project process but there are also organizations with multiproject processes i.e. managing several projects at the same time. Needs for single and multiproject management skills in organizations have motivated us to research two approaches in developing time-sensitive linking and navigation support for training timebased project management.

The bottom-up approach in our context means (a) to describe temporal rules or link structures such as "if $t>t_{1}$ then link to doc ${ }_{1}$ or "if $t_{1}<t<t_{2}$ then link to docgroup $p_{1-2}$ according to Allen's interval relations and (b) to formalize temporal linking rules by means of expanding the multiple linking mechanism defined by the XLink specification with time attributes. In practice this means constructing links to project documents from different blocks and connectors of blocks of the project flowchart according to temporal rules, which describe the progress of the project, states of related documents and their time status transitions.

When the user activates a certain block in the flowchart he/she can give appropriate time-conditions to retrieve related documents or he/she can navigate in a flowchart according to different temporal views. A specific search command can for instance be, "give me all documents that must have for approval -status within one week" or "give me all documents that I am responsible for and that must have for approval -status within one week".

The bottom-up approach concentrates on constructing time-sensitive linking rules on the content base level. The approach is dependent on resources involved in a certain project and thus is very project-specific. If the organization needs tools for training time-based multiproject management, a set of common temporal rules between different projects as 
well as project specific rules can be identified. This motivated us to research an approach, which includes both aspects.

The top-down approach gives a possibility to describe temporal rules common to all projects and hence the time-analysis needs to be carried out only once, nor in connection with every project. XML Topics Maps (XTM) 1.0 specification provides syntax for describing resource independent topic maps. With topic maps an associative information structure, which is located outside that information can be created.

A topic map usually contains several overlapping levels, which are semantically cross-linked. The core of the XML Topic Maps (XTM) 1.0 specification is formed of topics, which represent the subjects the topic map is about. Topics (T) can be grouped in classes called topic types. A topic type is a category to which one given topic instance belongs. A topic may be linked to one or more information resources that are relevant to the topic in some way. Such resources are called occurrences (O) of the topic. Occurrences are information resources relevant to a topic. Topics can be related through some association (A) expressing given semantics. Just as topic and occurrences can be grouped according to type so can also associations between topics be grouped according to their type. Each topic that participates in an association plays a role in that association called the association role. It is important to note that topic associations are completely independent of whatever information resources may or may not exist or be considered as occurrences of those topics. The same topic map can be overlaid on different pools of information to provide different views to users at different points of time or during different time intervals (Figure 1). In our case pools of information are project repositories. 


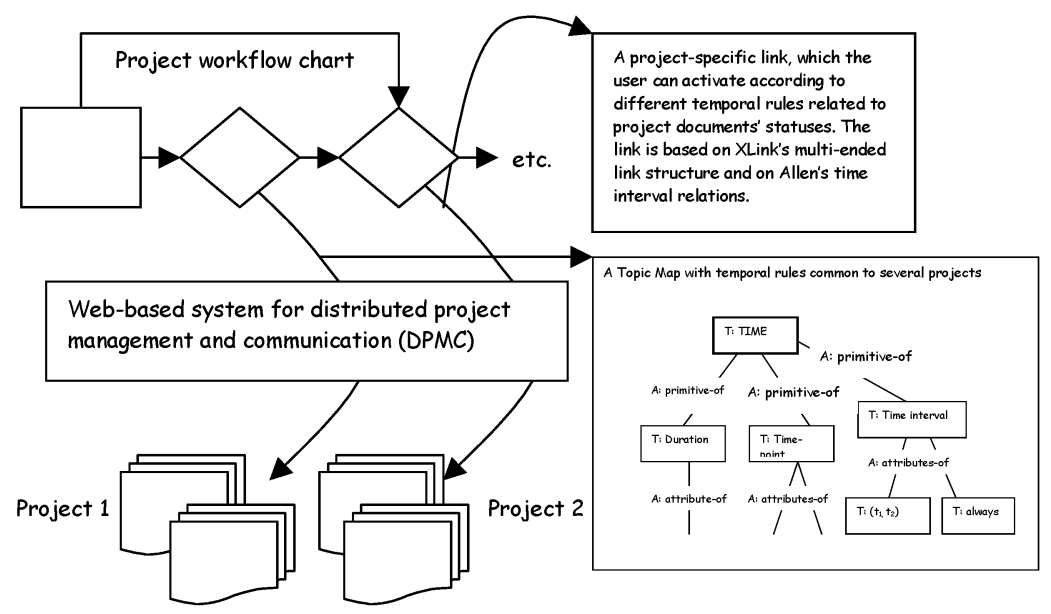

Multiproject documentspace

managed by the DPMC system on the Web

Figure 1. A project workflow chart with single and multiproject specific temporal hypertext linking and navigation support in distributed Web-based project management environment.

Our demonstration will be based on the Kronodoc environment (Kronodoc 2003) and on the flowchart presentation of the organization's information processes. The flowchart can be for instance a PowerPoint file and the links from the blocks or connectors of the blocks of the flowchart point to documents managed by the Kronodoc system. The construction of our demonstration will proceed in two phases.

1. we will add multiple temporal linking mechanisms to flowchart blocks.

2. We will investigate integrating an off-the-shelf Topic Map product such as Ontopia's Topic Map Engine or the K42 by Empolis to the flowchart (Empolis 2003; Ontopia 2003).

Already the first phase gives project management instructors new tools to illustrate and train time-based project management in organizations.

\section{CONCLUSIONS AND ISSUES FOR FURTHER RESEARCH}

In our paper we have proposed two approaches,a bottom-up approach based on the XLink language and a top-down approach based on the XTM specification, to develop dynamic workflow charts with time-sensitive linking and navigation support related to distributed project management and 
its training in organizations. We have presented our framework for demonstration. Although our paper has concentrated on developing tools for training time-based project management, the proposed approaches can be applied to other educational materials where flowchart presentations and time lines are essential such as in teaching history and natural sciences.

An interesting issue for further research is an ontology-driven topic maps approach. With this approach, the ontology is an explicit artifact distinct from the topic map it generates. One advantage of an ontology-driven topic map is its maintainability. When we create the ontology first from which the topic map will be generated we can separate the ontology design from the XTM implementation details. The ontology-driven topic maps approach makes it also possible to use existing ontologies instead of significant investment for creating topic maps from the very beginning.

\section{REFERENCES}

Allen, J. F. (1991). Time and time again: The many ways to represent time. International Journal of Intelligent Systems, 6(4), 341-355.

Berners-Lee, T., Hendler, J. \& Lassila, O. (2001). The Semantic Web. Scientific American, 284(5), 28-37.

Bieber, M., Vitali, F., Ashman, H., Balasubramanian, V. \& Oinas-Kukkonen, H. (1997). Fourth generation hypermedia: some missing links for the World Wide Web. International Journal of Human-Computer Studies, 47(1), 31-65.

Carr, L., Bechhofer, S. Goble, C. \& Hall, W. (2001). Conceptual linking: Ontology-based open hypermedia. In V. Shen, N. Saito, M. Lyu, \& M. Zurko (Eds.), Proceedings of the Tenth International Conference on World Wide Web. (pp. 334 -342). New York: ACM

Carr. L., Hall, W. \& DeRoure, D. (2000). The evolution of hypertext link services. ACM Computing Surveys. 31(4), $1-6$.

Empolis. (2003). Empolis Home Page (accessed 24.1.2003). http://www.empolis.co.uk.

Grønbæk, K. \& Trigg, R. H. (1999). From Web to workplace: Designing open hypermedia systems. Boston: MIT Press.

Guarino, N. (1995). Formal ontology, conceptual analysis and knowledge representation. International Journal of Human and Computer Studies, 43(5/6), 625 - 640.

Hameri, A. P. \& Heikkilä, J. (2002). Improving efficiency: Time-critical interfacing of project tasks. International Journal of Project Management. 20(2), 143-153.

Hameri, A. P. \& Nihtilä, J. (1997). Distributed new product development project based on Internet and World-Wide Web: A case study. The Journal of Product Innovation Management. 14(2) 77-87.

Hameri, A. P. \& Nihtilä, J. (1998). Data-based learning in product development. Scandinavian Journal of Management. 14(3), 223-238.

Heimbürger, A. (2003). Modelling time-sensitive linking mechanisms. In H. Jaakkola, H. Kangassalo, E., Kawaguchi, \& B. Thalheim (Eds.). Frontiers in Artificial Intelligence and Applications, Vol. 94: Information Modelling and Knowledge Bases XIV. (pp. 26-42). Amsterdam: IOS Press. 
ISO 13250. (2000). Information technology - SGML applications - Topic Maps. Geneva: International Organization for Standardization.

Kronodoc (2003). Kronodoc Home Page (accessed 11.2.2003) http//www.kronodoc.com.

Ontopia (2003). Ontopia Home Page (accessed 24.1.2003) http://www.ontopia.com.

Park, J. \& Hunting, S. (eds.) (2003). XML Topic Maps. Creating and using Topic Maps for the Web. Boston: Addison-Wesley.

Pepper, S. (2003). The TAO of Topic Maps. Finding the way in the age of infoglut. (accessed 18.1.2003) http://www.ontopia.net/topicmaps/materials/tao.html/.

Pepper, S. \& Garshol, L. M. (2003). The XML Papers. Lessons on applying Topic Maps. (accessed 24.1.2003) http://www.ontopia.net/topicmaps/materials/xmlconf.html/.

RosettaNet (2003). RosettaNet Home Page (accessed 23.1.2003) http://www.rosettanet.org/.

van Ossenbruggen, J., Hardman, L. \& Rutledge, L. (2002). Hypermedia and the Semantic Web: A research agenda. Journal of Digital Information, 3(1) [online], (accessed 27.1.2003) http://jodi.ecs.soton.ac.uk/Articles/v03/i01/VanOssenbruggen/.

W3C (2003a). The World Wide Web Consortium: Semantic Web Activity, (accessed 23.1.2003) http://www.w3.org/2001/sw/.

W3C (2003b). The World Wide Web Consortium: Extensible Markup Language (XML), (accessed 14.5.2003) http://www.w3.org/XML/.

W3C (2003c). The World Wide Web Consortium: XML Linking Language (XLink) Version 1.0 W3C Recommendation 27 June 2001, (accessed 23.1.2003) http://www.w3.org/TR/xlink/.

WordNet (2003). WordNet Home Page (accessed 23.1.2003) $\mathrm{http}: / /$ www.cogsci.princeton.edu/ wn/

XTM TopicMaps.Org. (2003). XML Topic Maps (XTM) 1.0. TopicMaps.Org Specification. (accessed 18.1.2003) http://www.topicmaps.org/xtm/1.0/

Zhou, Q. \& Fikes, R. (2002). A reusable time ontology (accessed 18.1.2003) http://www.ksl.stanford.edu/KSL_Abstracts/KSL-00-01.html/

\section{BIOGRAPHY}

Anneli Heimbürger is working as a senior research scientist at the Tampere University of Technology, Pori. Her research interests are in hypertext link management and XLinklanguage on the World Wide Web environment. Recent research has focused on developing time-sensitive linking and navigation tools and their applications. Her email address is anneli.heimburger@tut.fi. 